\title{
Toroidal formulation of
}

\section{nonlinear-rf-driven flows}

\author{
J. R. Myra and D. A. D'Ippolito \\ Lodestar Research Corporation
}

April 2002

\section{LODESTAR RESEARCH CORPORATION 2400 Central Avenue Boulder, Colorado 80301}




\title{
Toroidal formulation of nonlinear-rf-driven flows
}

\author{
J. R. Myra, D. A. D’Ippolito \\ Lodestar Research Corp., 2400 Central Ave. P-5, Boulder, Colorado 80301
}

\begin{abstract}
The nonlinear forces generated by localized rf absorption near a resonance layer can be employed to drive flows in a tokamak plasma. The present paper extends previous work to enable i) an evaluation of localized (in radius) poloidal and toroidal forces caused by rf absorption, ii) a rigorous calculation of the resulting poloidal and toroidal flow rates in the presence of damping due to plasma viscosity and a phenomenological radial diffusion of momentum, and iii) implicit treatment of ambipolarity issues in the presence of rf-generated forces. A rigorous result for the steady state poloidal flow that results from balancing plasma viscosity with the rf forces is obtained. Numerical results are presented for the case of ion Bernstein wave (IBW) interaction within a narrow resonant ion-cyclotron layer where the wave absorption and rf forces occur.
\end{abstract}

PACS: 52.50.Qt, 52.35.Mw, 52.30.-q, 52.55.Fa 


\section{Introduction}

The global response of the background plasma to applied rf may be fully characterized by the following quantities: the rf heating on each species, the three components of rf force, the driven parallel current, and the rf-induced fluxes of density and energy. These terms can be incorporated into the global equations for energy, density, poloidal and toroidal flows, Ohm's law, and the equilibrium equations for the rf-induced flux surface distortion. Heating and current drive have long been the subject of investigations and there has been some work on rf-induced fluxes. ${ }^{1}$ Here, we deal primarily with the question of how the rf-induced forces modify the background flows.

Because sufficiently large rf-driven sheared flows can in principle suppress turbulence, ${ }^{2}$ it has been proposed that $\mathrm{rf}$ power may be useful as an external control for triggering tokamak plasma transitions into high-confinement regimes. Indeed there is intriguing experimental evidence for this in ion Bernstein wave (IBW) experiments on a variety of tokamaks. ${ }^{3-7}$. Although the power was not sufficient to cause turbulence suppression in the IBW experiments on the Tokamak Fusion Test Reactor ${ }^{8}$ (TFTR), a measurement of the driven flows was possible. Understanding these experimental results remains a challenge for theory and modeling. In addition to the practical possibility of controlling turbulence, rf waves may provide a means of doing experiments on the physics of sheared flow generation that would be of interest to the turbulence community.

There is a rapidly growing literature on the theory and computation of nonlinear rf-induced flows. 9-17 The present paper follows most closely as an extension of work along the lines of Refs. 11-16 which, except for Ref. 12, treated the poloidal flows driven by ponderomotive-like forces in a slab geometry. Viscous damping of the flows was included heuristically in these calculations. The formalism for calculating the local rfinduced forces on the plasma is relatively well developed in these and other papers, ${ }^{18-20}$ and in references within them. In the present calculations, the rf force results are employed in the form given in Ref. 11. The goal of the present paper is to incorporate these rf calculations into a macroscopic description of a toroidal plasma. Two unique features of nonlinear rf-induced forces are that they can occur in a extremely narrow resonance layer, and that they can either input net momentum or simply redistribute momentum in a conservative way.

We do not consider the problem of rotation driven by energetic tail ions, which can absorb wave momentum and transport it by large orbit excursions and direct loss. 
This problem has been treated by other authors. ${ }^{21-25}$ Other rf-related mechanisms have also been proposed. 26 Instead, the mechanism for rotation considered here is closely related to the local ponderomotive force of the waves, suitably generalized to include the important concept of an rf-induced pressure tensor (a kind of rf-induced gyro-viscosity) and dissipation through cyclotron and Landau damping.

In the heuristic treatments of viscous flow damping referred to above, the poloidal force $\mathrm{F}_{\theta}$ and flow $\mathrm{u}_{\theta}$ are considered to be related by

$$
\rho \gamma_{\theta} u_{\theta}=F_{\theta}
$$

where $\rho$ is the mass density and $\gamma_{\theta}$ is a damping coefficient. Here, we work in orthogonal flux coordinates $(\psi, \theta, \zeta)$ corresponding to the radial, poloidal and toroidal directions respectively. Several objections may be made to Eq. (1). Most obviously, one needs to supply the damping rate $\gamma_{\theta}$ from a separate calculation. Moreover, examining the poloidal component of the steady-state momentum equation on which Eq. (1) is based leads to other concerns. The full equation is

$$
\mathbf{e}_{\theta} \cdot \nabla \cdot \Pi=\mathrm{F}_{\theta}-\frac{1}{\mathrm{c}} \mathrm{J}_{\psi} \mathrm{B}_{\zeta}-\frac{\mathrm{B}}{\mathrm{B}_{\theta}} \nabla_{\|} \mathrm{p}
$$

where $\Pi$ is the viscosity tensor and other symbols (to be defined precisely later) have their usual meanings. The damping rate $\gamma_{\theta}$ arises from $\Pi$, but the heuristic result does not retain any rf-driven ambipolar current $\mathrm{J}_{\psi}$ or possible rf-induced poloidal variations of pressure. These objections can be circumvented by employing a proper flux surface average of the parallel component instead of the poloidal component (as will be done subsequently), but the way in which the driving term for poloidal flows is transformed from $F_{\|}$to $F_{\theta}$ is important to understand.

The goal of this paper is to develop a formalism to clarify these issues and to apply the formalism to calculate the flows induced by the absorption of an IBW. The formalism will include viscous flow damping, radial diffusion, and ambipolarity considerations in the presence of $\mathrm{rf}$. The latter have previously been considered for the slab geometry case by a more direct approach. ${ }^{14}$ Some background for the treatment of the toroidal aspects of the present calculation may be found in Refs. 17 and 27-29.

The plan of our paper is as follows. In Sec II we develop the basic formulation of toroidal and poloidal flows and explore several limiting cases of interest for the nonlinear rf-induces forces. The section ends with an explicit representation for the rf forces. Sec. III gives sample numerical results for the poloidal and toroidal flows driven by an 
IBW that is absorbed in an ion cyclotron resonance layer. An approximate analytical scaling of the resulting flows is also obtained. A summary of our main results is given in Sec. IV.

\section{Derivation}

\section{A. Toroidal formulation}

We begin with the species summed momentum equation for the evolution of the background plasma

$$
\frac{\partial}{\partial \mathrm{t}}(\rho \mathbf{u})+\nabla \mathrm{p}+\nabla \cdot \Pi=\mathbf{F}+\frac{1}{\mathrm{c}} \mathbf{J} \times \mathbf{B}
$$

where $\mathrm{p}=\mathrm{n}\left(\mathrm{T}_{\mathrm{i}}+\mathrm{T}_{\mathrm{e}}\right)$ is the pressure, $\Pi$ is the traceless plasma viscosity tensor (not to be confused with the rf pressure tensor ${ }^{11}$ that contributes to $\left.\mathbf{F}\right), \rho=\mathrm{nm}_{\mathrm{i}}$, and $\mathbf{F}$ is the total nonlinear force of the $\mathrm{rf}$ waves on the plasma (including the contributions of the $\mathrm{rf}$ pressure tensor and the time-averaged rf Lorentz force). The rf force $\mathrm{F}$ will be given explicitly in Sec. II D.

The steady state toroidal and parallel momentum equations are

$$
\begin{gathered}
\mathrm{Re}_{\zeta} \cdot \nabla \cdot \Pi=\mathrm{RF}_{\zeta}+\frac{1}{\mathrm{c}} \mathrm{J}_{\psi} \mathrm{RB}_{\theta} \\
\mathbf{B} \cdot \nabla \cdot \Pi=\mathrm{BF}_{\|}-\mathrm{B} \nabla_{\| \mathrm{p}}
\end{gathered}
$$

where $\mathrm{R}$ is the major radius and axisymmetry has been employed. When a flux surface average [see Eqs. (9) and (10)] is performed on the above equations, it can be shown that many of the terms are annihilated:

$$
\left\langle\mathrm{J}_{\psi} \mathrm{RB}_{\theta}\right\rangle=\left\langle\mathrm{B} \nabla_{\| \mathrm{p}}\right\rangle=0
$$

where we have used $\nabla \cdot \mathbf{J}=0$, and note that for any quantity $\mathrm{Q},\left\langle\mathrm{B} \nabla_{\|} \mathrm{Q}\right\rangle=0$. When only lowest order neoclassical processes are considered, we also have

$$
\left\langle\operatorname{Re}_{\zeta} \cdot \nabla \cdot \Pi\right\rangle=\left\langle\nabla \cdot\left(\Pi \cdot \operatorname{Re}_{\zeta}\right)\right\rangle=\frac{1}{v} \frac{\partial}{\partial \psi} v\left\langle\mathrm{R}^{2} \mathrm{~B}_{\theta} \Pi_{\psi \zeta}\right\rangle=0
$$

where $\Pi$ is a symmetric tensor. Thus, we are motivated to introduce a phenomenological diffusion of momentum to balance the force term in the toroidal equation. For the case of nonlinear rf-induced flows considered here, where the force is typically localized in a 
narrow rf resonant layer, we introduce a phenomenological diffusion of momentum in both the toroidal and parallel equations. This allows treatment of the case where the rf deposition layer (forcing layer) is sufficiently narrow that diffusion can dominate the viscosity in the parallel equation. As we shall see, diffusion impacts the conceptual understanding of the flow drive. The flux-surface averaged equations including diffusion are:

$$
\begin{gathered}
-\frac{\mathrm{D}}{v} \frac{\partial}{\partial \psi} v\left\langle\mathrm{R}^{2} \mathrm{~B}_{\theta}^{2} \frac{\partial}{\partial \psi} \rho \mathrm{Ru}_{\zeta}\right\rangle=\left\langle\mathrm{RF}_{\zeta}\right\rangle \\
-\frac{\mathrm{D}}{v} \frac{\partial}{\partial \psi} v\left\langle\mathrm{R}^{2} \mathrm{~B}_{\theta}^{2} \frac{\partial}{\partial \psi} \rho \mathrm{Bu}_{\|}\right\rangle+\langle\mathbf{B} \cdot \nabla \cdot \Pi\rangle=\left\langle\mathrm{BF}_{\|}\right\rangle
\end{gathered}
$$

where $\mathbf{J}=\partial(\mathrm{x}, \mathrm{y}, \mathrm{z}) / \partial(\psi, \theta, \zeta)$ is the Jacobian and the flux surface average for any quantity $\mathrm{Q}$ is defined by

$$
\begin{gathered}
\langle Q\rangle=\frac{1}{v} \int \frac{d \zeta}{2 \pi} \int d \theta J Q \\
v=\int d \theta J .
\end{gathered}
$$

Next, we employ the flow representation in an axisymmetric torus as

$$
\mathbf{u}=\mathrm{K}(\psi) \mathbf{B}+\mathrm{G}(\psi) \operatorname{Re}_{\zeta}
$$

This form insures several important properties of the flow, including incompressibility (which requires $\mathrm{K}$ to be a flux function) the absence of differential rotation within a flux surface (which requires $\mathrm{G}$ to be a flux function), and hence no "winding up" of the Bfield. 30

The radial force balance equation gives

$$
\mathrm{G}=-\mathrm{c}\left(\frac{\partial \Phi}{\partial \psi}+\frac{1}{\operatorname{Zen}} \frac{\partial \mathrm{p}_{\mathrm{i}}}{\partial \psi}\right)+\frac{\mathrm{c}}{\mathrm{Zen}_{\mathrm{i}}} \frac{\mathrm{F}_{\mathrm{i} \psi}}{\mathrm{RB}_{\theta}}
$$

This equation shows that there are three ways the $\mathrm{rf}$ can affect $\mathrm{E}_{\mathrm{r}} \propto \partial \Phi / \partial \psi$ : (i) by the toroidal rotation it drives, i.e. through $\mathrm{G}$ (the relationship of $\mathrm{G}$ to the $\mathrm{rf}$ fields is given later); (ii) by the $\mathrm{p}_{\mathrm{i}}(\psi)$ heating profile; and (iii) directly through the radial ponderomotive force term $\mathrm{F}_{\mathrm{i} \psi} \cdot{ }^{17}$ Often $\mathrm{F}_{\mathrm{i} \psi}$ is small compared with the other terms in the radial force balance equation, but if not, the rf can also drive flux surface distortions. ${ }^{31}$ 


\section{B. Flows in the limit of rapidly varying $K$ and $G$}

Assuming the radial variation of $\mathrm{K}$ and $\mathrm{G}$ is much more rapid (i.e. on shorter scales) than $B, v$ and $\rho$, we can introduce a local radial variable $x$ in the vicinity of the rf resonance layer with

$$
\left\langle\mathrm{R}^{2} \mathrm{~B}_{\theta}^{2}\right\rangle \mathrm{dx} \mathrm{x}^{2}=d \psi^{2}
$$

and rewrite the toroidal and parallel equations as

$$
\begin{gathered}
-\mathrm{D} \rho \mathrm{RB} \mathrm{K} \mathrm{K}^{\prime \prime}-\mathrm{D} \rho\left\langle\mathrm{R}^{2}\right\rangle_{\mathrm{x}} \mathrm{G}^{\prime \prime}=\left\langle\mathrm{RF}_{\zeta}\right\rangle \\
-\mathrm{D} \rho\left\langle\mathrm{B}^{2}\right\rangle_{\mathrm{x}} \mathrm{K}^{\prime \prime}-\mathrm{D} \rho \mathrm{RB}_{\zeta} \mathrm{G}^{\prime \prime}+3 \eta_{0} \mathrm{~K}\left\langle\left(\nabla_{\|} \mathrm{B}\right)^{2}\right\rangle=\left\langle\mathrm{B} F_{\|}\right\rangle
\end{gathered}
$$

where ${ }^{\prime}=\mathrm{d} / \mathrm{dx}$, and for any quantity $\mathrm{Q}$,

$$
\langle\mathrm{Q}\rangle_{\mathrm{x}}=\frac{\left\langle\mathrm{R}^{2} \mathrm{~B}_{\theta}^{2} \mathrm{Q}\right\rangle}{\left\langle\mathrm{R}^{2} \mathrm{~B}_{\theta}^{2}\right\rangle}
$$

Although we shall omit the details, we have used the fact that in the PfirschSchlüter limit (strong collisionality)

$$
\langle\mathbf{B} \cdot \nabla \cdot \Pi\rangle=3 \eta_{0} \mathrm{~K}\left\langle\left(\nabla_{\|} \mathrm{B}\right)^{2}\right\rangle
$$

where $\eta_{0}=0.96 \mathrm{p} / v_{\mathrm{ii}}$ is the classical parallel ion viscosity. We take the Pfirsch-Schlüter limit in this section in order to have a concrete and tractable expression that illustrates the correct properties of $\Pi$; the formulation presented here is general and not restricted to this collisional regime. The fact that only K, not G, appears on the rhs of Eq. (17) implies (as is well known) that parallel viscosity damps poloidal rotation, but not purely toroidal rotation.

Equations (14) and (15) can be solved for K and G to yield

$$
\begin{gathered}
{\left[\left\langle\mathrm{B}^{2}\right\rangle_{\mathrm{x}}-\frac{\mathrm{R}^{2} \mathrm{~B}_{\zeta}^{2}}{\left\langle\mathrm{R}^{2}\right\rangle_{\mathrm{x}}}\right]^{\prime \prime}-\frac{3 \eta_{0}}{\mathrm{D} \rho}\left\langle\left(\nabla_{\|} \mathrm{B}\right)^{2}\right\rangle \mathrm{K}=-\frac{\left\langle\mathrm{B} F_{\|}\right\rangle}{\mathrm{D} \rho}+\frac{\mathrm{RB} \zeta\left\langle\mathrm{RF}_{\zeta}\right\rangle}{\mathrm{D} \rho\left\langle\mathrm{R}^{2}\right\rangle_{\mathrm{x}}}} \\
\mathrm{G}^{\prime \prime}=-\frac{\left.\langle\mathrm{RF}\rangle_{\zeta}\right\rangle}{\mathrm{D} \rho\left\langle\mathrm{R}^{2}\right\rangle_{\mathrm{x}}}-\frac{\mathrm{RB}_{\zeta} \mathrm{K}^{\prime \prime}}{\left\langle\mathrm{R}^{2}\right\rangle_{\mathrm{x}}} .
\end{gathered}
$$


A casual examination of Eq. (15) in the limit $\mathrm{D} \rightarrow 0$ might suggest that poloidal rotation (proportional to $\mathrm{K}$ ) is driven by the parallel component of the force. However, when the $\mathrm{D} \rightarrow 0$ limit is taken in Eq. (18) it is evident that the toroidal force is also present, and that the combination of forces appearing on the rhs of Eq. (18) is closely related to the poloidal force (and becomes it if the flux surface averages are ignored). It will be noted that when $\mathrm{D}=0$, Eq. (14) is ill-posed when $\mathrm{F}_{\zeta} \neq 0$ unless $\mathrm{DG}^{\prime \prime}$ is retained. Thus $\mathrm{DG}^{\prime \prime}$ remains finite in Eq. (15) and cannot be neglected.

The main point here is that retaining diffusion in the parallel momentum equation is important when considering the steady state flows. In the literature, diffusion is normally retained in the toroidal equation because there is no toroidal damping of flows. It is tempting, but incorrect, to drop diffusion in the steady state parallel equation when it is smaller than the damping term. Our analysis retaining $\mathrm{D}$ shows that the $\mathrm{D}=0$ case is singular because then $\mathrm{G} \rightarrow \infty$. Furthermore, when $\mathrm{D}$ is retained, the poloidal flow $\mathrm{K}$ is seen to be driven by a combination of flux surfaced averaged forces that resembles $F_{\theta}$, as in the heuristic model, not $\mathrm{F}_{\|}$. Evidently, the combination of terms that appears on the rhs of Eq. (18) is just what is required to account for the $J_{\psi}$ and $\nabla_{\|}$p terms in Eq. (2).

\section{Flows in the circular flux surface limit}

\section{Basic equations}

In this section, we return to Eqs. (7) and (8) (before the assumption of rapid variations of $\mathrm{K}$ and $\mathrm{G}$ was made) and consider the circular flux surface limit. Expanding for small $\varepsilon=r / R_{0}$ with $\mathrm{R}=\mathrm{R}_{0}(1+\varepsilon \cos \theta)$ and working through order $\varepsilon^{2}$ we obtain

$$
\begin{gathered}
\mathrm{D}_{\mathrm{r}}^{2}\left[\rho \mathrm{KI}(\psi)+\rho \mathrm{GR}_{0}^{2}\left(1+\frac{3 \varepsilon^{2}}{2}\right)\right]=-\left\langle\mathrm{RF}_{\zeta}\right\rangle \\
\mathrm{D} \nabla_{\mathrm{r}}^{2}\left[\rho \mathrm{K} \frac{\mathrm{I}(\psi)^{2}}{\mathrm{R}_{0}^{2}}\left(1+\mathrm{b}_{\theta}^{2}+\frac{\varepsilon^{2}}{2}\right)+\rho \mathrm{GI}(\psi)\right]-\langle\mathbf{B} \cdot \nabla \cdot \Pi\rangle=-\left\langle\mathrm{BF}_{\|}\right\rangle
\end{gathered}
$$

where $\nabla_{\mathrm{r}}^{2}$ is the usual cylindrical Laplacian operator, $\rho, \mathrm{K}, \mathrm{G}, \mathrm{q}$, and $\mathrm{I}(\psi)=\mathrm{RB}_{\zeta}$ are flux functions, $\mathbf{b}=\mathbf{B} / \mathrm{B}, \mathrm{b}_{\theta} \approx \varepsilon / \mathrm{q}, \mathrm{b}_{\zeta} \approx 1-\varepsilon^{2} / \mathrm{q}^{2}$ and it is useful to note that for any quantity $\mathrm{Q},\langle\mathrm{Q}\rangle=\int \mathrm{d} \theta / 2 \pi(1+\varepsilon \cos \theta) \mathrm{Q},\left\langle\mathrm{B}^{2}\right\rangle=\left(\mathrm{I} / \mathrm{R}_{0}\right)^{2}\left(1+\varepsilon^{2} / \mathrm{q}^{2}+\varepsilon^{2} / 2\right)$ and $\left\langle\mathrm{R}^{2}\right\rangle=\mathrm{R}_{0}^{2}(1$ $\left.+3 \varepsilon^{2} / 2\right)$. Defining the auxiliary quantities $\hat{\mathrm{J}}, \hat{\mathrm{K}}$ and $\Gamma$ by

$$
\hat{\mathbf{J}}=\rho \mathrm{GR}_{0}\left(1+3 \varepsilon^{2} / 2\right)+\rho \mathrm{KB}_{\zeta_{0}}=\rho \mathrm{u}_{\zeta}
$$




$$
\begin{aligned}
& \hat{\mathrm{K}}=\rho \mathrm{KB}=\rho u_{\theta} / \mathrm{b}_{\theta} \\
& \Gamma \hat{\mathrm{K}} \mathrm{B}=<\mathbf{B} \cdot \nabla \cdot \Pi>
\end{aligned}
$$

Eqs. (20) and (21) may be combined into the form

$$
\begin{gathered}
\mathrm{D} \nabla_{\mathrm{r}}^{2}(\hat{\mathrm{J}})=-\mathrm{F}_{\zeta} \\
\mathrm{D} \nabla_{\mathrm{r}}^{2}\left[\mathrm{~b}_{\theta}^{2} \hat{\mathrm{K}}\left(1+2 \mathrm{q}^{2}\right)\right]-\Gamma \hat{\mathrm{K}}=-\mathrm{b}_{\theta} \mathrm{F}_{\theta}
\end{gathered}
$$

where $\left\langle\mathrm{RF}_{\zeta}\right\rangle \equiv \mathrm{R}_{0} \mathrm{~F}_{0 \zeta},\left\langle\mathrm{BF}_{\|}>\equiv \mathrm{I}(\psi) \mathrm{F}_{0 \|} / \mathrm{R}_{0}, \mathrm{~b}_{\theta} \mathrm{F}_{0 \theta}=\mathrm{F}_{0 \|}-\mathrm{F}_{0 \zeta}\right.$ and henceforth in this section we abbreviate $\mathrm{I}(\psi) / \mathrm{R}_{0}=\mathrm{B}_{\zeta 0} \approx \mathrm{B}$ where only lowest order information is required; and we also abbreviate $\mathrm{F}_{0 \zeta}, \mathrm{F}_{0 \theta}$ and $\mathrm{F}_{0 \|}$ by $\mathrm{F}_{\zeta}, \mathrm{F}_{\theta}$ and $\mathrm{F}_{\|}$respectively.

Equations (25) and (26) are the principal results of this subsection. To make analogy with Eq. (1), we can define

$$
\gamma_{\theta}=\Gamma / b_{\theta}^{2}
$$

\section{Viscous damping limit: small D}

We first consider the limit of small D such that $\mathrm{D} / \Delta^{2}<<\gamma_{\theta}$ or $\lambda \ll \Delta$, where $\Delta$ is the characteristic radial scale length of the rf forces $F_{\theta}$ and $F_{\|}$(i.e. the width of rf absorption layer) and

$$
\lambda=\left(\frac{\mathrm{D}\left(1+2 \mathrm{q}^{2}\right)}{\gamma_{\theta}}\right)^{1 / 2}
$$

is the length scale at which diffusion and damping compete. In this limit the diffusion term may be neglected in Eq. (26) and the result for the poloidal flow has the form of the heuristic result of Eq. (1) with the effective damping rate given by

$$
\gamma_{\theta}=\frac{3 v_{i}^{2}}{2 R^{2} v_{i i}}
$$

in the Pfirsch-Schlüter regime.

It is important to note that $\gamma_{\theta}$ is not the rate at which an initialized flow damps in time. We can recover the standard result for the time-dependent damping rate ${ }^{32}$ by reinstating the time derivatives and dropping the forcing terms to obtain (after some algebra) 


$$
\gamma_{t}=\frac{3 \eta_{0}}{2 \rho R^{2}\left(1+2 q^{2}\right)}=\frac{3 v_{i}^{2}}{2 v_{i i} R^{2}\left(1+2 q^{2}\right)}
$$

The extra factor $\left(1+2 q^{2}\right)$, familiar from the MHD analysis of slowly growing modes, is an inertial term resulting from parallel flows that does not enter the steady state (forced) $\mathrm{D}=0$ results.

\section{Diffusion limit: larger $D$}

Typically, the rf-layer width is narrow and the calculation must be done in the diffusion limit $\mathrm{D} / \Delta^{2}>>\gamma_{\theta}$. First we consider the case $\Delta<<\lambda<<r_{\mathrm{a}}$ where $\mathrm{r}_{\mathrm{a}}$ is the minor radius. In this limit the poloidal flow is localized, but due to diffusion the flow layer is broader than the $\mathrm{rf}$ absorption layer. The analysis proceeds by approximating the localized forces by a delta-function at the rf absorption layer. The equation for $\mathrm{K}$ has the form

$$
a K^{\prime \prime}-b K=S
$$

where the exact definitions of a, b, and S are evident by comparison with Eq. (26) and are of order

$$
\begin{gathered}
\mathrm{a} \approx \mathrm{B}_{\theta}^{2} \\
\mathrm{~b} \approx-\frac{3 \eta_{0} \mathrm{~B}^{2} \mathrm{r}^{2}}{2 \mathrm{D \rho q} \mathrm{R}^{4}} \\
\mathrm{~S} \equiv \mathrm{S}_{0} \delta\left(\mathrm{x}-\mathrm{x}_{0}\right) \approx-\frac{\mathrm{B}_{\theta} \mathrm{F}_{\theta}}{\mathrm{D} \rho}
\end{gathered}
$$

In the force-free region, the solution for $\mathrm{K}$ is

$$
\mathrm{K}=\mathrm{K}_{0} \mathrm{e}^{ \pm\left(\mathrm{x}-\mathrm{x}_{0}\right) / \lambda}
$$

where $\lambda=(a / b)^{1 / 2}$. The jump condition across $x_{0}$ corresponding to Eq. (31) is

$$
\mathrm{a}\left[\frac{\mathrm{dK}}{\mathrm{dx}}\right]_{-}^{+}=\int \mathrm{dx} S
$$

which yields

$$
\mathrm{K}_{0}=-\frac{\lambda}{2 \mathrm{a}} \int \mathrm{d} \mathrm{x} S
$$


This result yields a peak value for $\mathrm{K}$ that is smaller than in the dampingdominated limit (where $\mathrm{K}=-\mathrm{S} / \mathrm{b}$ ) by the factor $\Delta / 2 \lambda$ where $\Delta$ is the $\mathrm{rf}$ deposition layer width. The size of maximum poloidal flow scales like $u_{\theta} \propto 1 / D$. The poloidal flow layer width $\lambda \propto \mathrm{D}^{1 / 2}$ is also larger in the present limit $(\lambda \gg \Delta)$ than in the damping-dominated case $(\lambda<\Delta)$ so the shear in the poloidal flow is further reduced by comparison.

The toroidal flow is always diffusion dominated since there is no toroidal damping in our model. Thus, the present limit leads to global toroidal flows.

\section{Strong diffusion limit}

The strong diffusion limit is defined by $\Delta<<\lambda \sim \mathrm{r}_{\mathrm{a}}$. In this case, both the poloidal and toroidal flows are global, and the diffusion scale includes the entire minor radius. Net momentum input $\int d x F_{\theta}$ and $\int d x F_{\zeta}$ drives net flows, but net flows are also possible with no direct rf momentum input. This fact has been noted in the context of rotation driven by energetic tail ions. 22 Here, it may be seen by considering a. "dipole" forcing term, e.g. $\mathrm{F}_{\zeta}$ or $\mathrm{F}_{\theta} \propto \delta^{\prime}\left(\mathrm{x}-\mathrm{x}_{0}\right)$. As in Ref. 22 , the boundary conditions or some other symmetry-breaking mechanism are critical to the result. Here it could be simply different boundary conditions at $r=0$ and $r=r_{a}$, or different $D$ on either side of the shear-flow layer. The latter might arise, for example, as a natural consequence of the effect of sheared flows on the turbulence.

\section{Explicit representation of rfforces}

To proceed further, an explicit representation of the $\mathrm{rf}$ forces is required. A general eikonal result has been obtained in Ref. 11 and will be summarized here. The result is valid for arbitrary electromagnetic waves in a hot plasma.

The nonlinear force density of rf waves on a fluid element is given by

$$
\mathbf{F}=\frac{1}{16 \pi}\left[\left(\nabla \mathbf{E}^{*}\right) \cdot \mathbf{P}-\nabla \cdot\left(\mathbf{P E}^{*}\right)+\mathrm{cc}\right]-\nabla \cdot \Pi_{\mathrm{rf}}
$$

where $\mathbf{P}=\chi \cdot \mathbf{E}, \chi$ is the dielectric susceptibility tensor and $\Pi_{\mathrm{rf}}$ is the $\mathrm{rf}$ pressure tensor. The off-diagonal terms of $\Pi_{\mathrm{rf}}$ are sufficient to describe the momentum redistribution forces due to hot plasma ion gyroradius effects. It was shown ${ }^{11}$ that these terms can be expressed in terms of the rf-linearized distribution function $\mathrm{f}_{1}$ 


$$
\begin{array}{r}
\Pi_{\mathrm{rf}}=\frac{\mathrm{m}}{4 \Omega} \int \mathrm{d}^{3} \mathrm{vf}_{1}\left[\frac{1}{4}\left(\mathbf{a}^{*} \mathbf{v} \times \mathbf{b}+\mathbf{v a} \mathbf{a}^{*} \times \mathbf{b}\right)+\right. \\
\left.\frac{3}{4}\left(\mathbf{a}_{\|}^{*} \mathbf{v} \times \mathbf{b}+\mathbf{v}_{\|} \mathbf{a}^{*} \times \mathbf{b}\right)\right]+\mathrm{tr}+\mathrm{cc}
\end{array}
$$

where $\operatorname{tr}$ is the transpose and $\mathbf{a}=\mathrm{Ze} / \mathrm{m}\left(\mathbf{E}_{1}+\mathbf{v} \times \mathbf{B}_{1} / \mathrm{c}\right)$ is the linearized acceleration of the particles in the wave fields. Equations (38) and (39) are appropriate for studying the rfinduced flows from a full 2D toroidal wave code. Such a study will be deferred to a later paper. For present purposes, several characteristic features of rf-induced flows can be illustrated by taking the rf-fields from an appropriately rotated 1D slab model local to the rf resonance layer. The resulting forces in the flux surface for a case with rf field gradients in the $\mathrm{x}$ direction are

$$
\begin{gathered}
\mathrm{F}_{\mathrm{y}}=\frac{\mathrm{k}_{\mathrm{y}}}{4 \omega}\left(\mathbf{E}^{*} \cdot \mathbf{J}\right)-\frac{\partial}{\partial \mathrm{x}}\left[\frac{\mathrm{i}}{4 \omega} \mathrm{J}_{\mathrm{x}} \mathrm{E}_{\mathrm{y}}{ }^{*}+\frac{1}{8 \Omega}\left(\mathrm{J}_{\mathrm{y}} \mathrm{E}_{\mathrm{y}}{ }^{*}-\mathrm{J}_{\mathrm{x}} \mathrm{E}_{\mathrm{x}}{ }^{*}\right)\right]+\mathrm{cc} \\
\mathrm{F}_{\mathrm{z}}=\frac{\mathrm{k}_{\mathrm{z}}}{4 \omega}\left(\mathbf{E}^{*} \cdot \mathbf{J}\right)-\frac{\partial}{\partial \mathrm{x}}\left[\frac{\mathrm{i}}{4 \omega} \mathrm{J}_{\mathrm{x}} \mathrm{E}_{\mathrm{z}}{ }^{*}+\frac{1}{4 \Omega}\left(\mathrm{J}_{\mathrm{z}} \mathrm{E}_{\mathrm{y}}{ }^{*}+\mathrm{J}_{\mathrm{y}} \mathrm{E}_{\mathrm{z}}{ }^{*}\right)\right]+\mathrm{cc}
\end{gathered}
$$

where $\mathbf{e}_{\mathrm{x}}=\mathbf{e}_{\psi}, \mathbf{e}_{\mathrm{z}}=\mathbf{b}$ and $\mathbf{e}_{\mathrm{y}}=\mathbf{b} \times \mathbf{e}_{\psi}$.

There are two classes of terms which contribute to the forces. The terms proportional to the components of $\mathbf{k}\left(\mathbf{E}^{*} \cdot \mathbf{J}\right)$ correspond to direct absorption of wave momentum $\mathbf{k} / \omega$ by the plasma for each "quantum" of absorbed wave energy. The remaining terms, which are perfect derivatives in $\mathrm{x}$, correspond to a conservative redistribution of momentum.

Given the field line geometry, the $\theta$ and $\zeta$ components of the force needed for the flow calculations can be determined by projection.

\section{An example of IBW driven flows}

The IBW-driven flows observed on TFTR $^{8}$ provide a well-diagnosed and modeled case $^{15,33}$ on which to apply the present formalism. Here we consider an IBW incident on the $\omega=5 \Omega_{\mathrm{T}}$ resonance, with parameters $\mathrm{k}_{\mathrm{y}}=0$ and $\mathrm{k}_{\mathrm{z}}=5 \mathrm{~m}^{-1}$. Since the resonance layer is narrow compared to the plasma cross-section, the wave fields are computed by a onedimensional ray tracing analysis 12 which employs the Bessel and Z-function ion response in the resonance layer. The results given here employ a simple single resonance model for a pure $\mathrm{T}$ plasma in the electrostatic limit for purposes of illustration. More detailed studies of this problem in a DT plasma with all the Bessel functions sums and full field 
polarization 15 yield an essentially similar result for the wave fields, and comparisons have shown that the eikonal and full spectral width calculations of the rf forces are equivalent for this case. ${ }^{34}$

\section{A. Numerical results}

Figure 1 shows the electric field $\mathrm{E}_{\mathrm{x}} \sim 100 \mathrm{~V} / \mathrm{cm}$, Poynting flux and absorbed power for a case with total power absorption $\sim 400 \mathrm{~kW}$. The wave enters the figure from the right. The electric field undergoes a large number oscillations between $\mathrm{x}=5$ and 2 $\mathrm{cm}$ before its amplitude becomes negligible, justifying the eikonal limit. The $\omega=5 \Omega_{\mathrm{T}}$ resonance is located at $\mathrm{x}=0$.

Figures 2 and 3 show the nonlinear forces and resulting toroidal and poloidal flows obtained by solving Eqs. (25) and (26) for the parameters $\gamma_{\theta}=10 / \mathrm{s}$ and $\mathrm{D}=200$ $\mathrm{cm}^{2} / \mathrm{s}$. The poloidal flow equation is singular on axis when $\mathrm{D}$ and $\gamma$ are radially constant; therefore, in the code the form of the equations near the axis is regularized to give finite flows. Note, in Fig. $2 b$, the bipolar nature of $F_{\theta}$ and the diffusive broadening of $u_{\theta}$ relative to $F_{\theta}$. The poloidal flow is in the diffusion limit. The bipolar structure is due to the fact that $\mathrm{k}_{\mathrm{y}}=0$ implies no net poloidal momentum input for this case. The peak value of $u_{\theta} \approx 2 \mathrm{~km} / \mathrm{s}$ (for this assumed D) is of the same order as that measured experimentally in TFTR $^{8}$ and the profile shape shows qualitative agreement with the experiment. However, we stress that no attempt at detailed modeling has been made here. While $\gamma_{\theta}$ is typical of the banana regime value that is expected to apply under these conditions, the correct value for the momentum diffusion coefficient $\mathrm{D}$ in this experiment is not known. The calculated toroidal flow velocity profile for this example is $\approx 30 \mathrm{~km} / \mathrm{s}$ at $\mathrm{r}=0$ and is approximately constant out to the resonance layer $(\mathrm{r}=46 \mathrm{~cm})$ after which it decays slowly to zero at $r=r_{a}=100 \mathrm{~cm}$, as shown in Fig. 3. It is global, and driven primarily by the net toroidal momentum of the waves, which were taken to have an asymmetric spectrum with $\mathrm{k}_{\mathrm{Z}} \sim 5 \mathrm{~m}^{-1}$.

\section{B. Analytical scaling of flows (diffusive limit)}

The poloidal flow for this example is consistent with the estimate

$$
\mathrm{u}_{\theta}=\mathrm{P}_{\mathrm{rf}} \Delta / 4 \mathrm{q}^{2} \Omega \mathrm{D} \rho \mathrm{V}
$$

where $\mathrm{V}=4 \pi^{2} \operatorname{Rr} \Delta$ is the relevant volume for localized flows. In terms of experimental inputs we have 


$$
\mathrm{u}_{\theta} \sim 0.4 \mathrm{P}_{\mathrm{rf}} / \mathrm{q}^{2} \mathrm{R} \text { r B D n } 20
$$

where the units are $\mathrm{u}_{\theta}(\mathrm{km} / \mathrm{s}), \mathrm{P}_{\mathrm{rf}}(\mathrm{MW}), \mathrm{R}(\mathrm{m}), \mathrm{r}(\mathrm{m}), \mathrm{B}(\mathrm{T}), \mathrm{D}\left(\mathrm{m}^{2} / \mathrm{s}\right)$, and $\mathrm{n}\left(10^{20} / \mathrm{m}^{3}\right)$.

For the toroidal flow, the estimate is

$$
\mathrm{u}_{\zeta}=\mathrm{P}_{\mathrm{rf}} \mathrm{k}_{\zeta} \mathrm{r}^{2} / \omega \mathrm{D} \rho \mathrm{V}
$$

where the relevant volume for global toroidal flows is now $\mathrm{V}=2 \pi^{2} \mathrm{Rr}^{2}$. This yields

$$
\mathrm{u}_{\zeta} \sim 48 \mathrm{P}_{\mathrm{rf}} \mathrm{k}_{\zeta} / \mathrm{RfD} \mathrm{n}_{20} \mu
$$

with the same units as for Eq. (43) and additionally $\mathrm{k}_{\zeta}\left(\mathrm{m}^{-1}\right)$, the ion mass $\mathrm{m}_{\mathrm{p}} / \mathrm{m}_{\mathrm{i}}=\mu(\mathrm{amu})$ and frequency $\mathrm{f}(\mathrm{MHz})$.

\section{Summary}

In this paper we have developed a formulation of rf-driven flows in toroidal geometry which implicitly includes rf ambipolarity effects. Three mechanisms have been identified by which rf affects the radial electric field $\mathrm{E}_{\mathrm{r}}$ and the flows [see Eq. (12)]: through heating $\left(\mathrm{p}_{\mathrm{i}}\right)$, explicit momentum input or redistribution (flow drive), and nonlinear radial forces. In this paper we have concentrated on the flow drive mechanism.

It was shown that retaining diffusion in the parallel momentum equation is critical in the derivation, and results in $\mathrm{u}_{\theta}$ being driven by $\mathrm{F}_{\theta}$ [exactly in the circular plasma limit of Eq. (26), and approximately in the general case of Eq. (18)] as expected from a heuristic treatment. In a rigorous calculation of the damping of poloidal flows by plasma viscosity in the Pfirsch-Schlüter regime, it was found that $\gamma_{\theta}$ given by Eq. (29) differs by the factor $\left(1+2 q^{2}\right)$ from the damping rate that appears in the time-dependent problem. Several diffusive regimes were identified for the poloidal flows giving rise to various degrees of poloidal flow localization. Toroidal flows are always global, even though the rf-driving terms are highly localized. The main theoretical results are contained in Eqs. (18) and (19) for evaluating the flows in a narrow rf layer in general geometry, and Eqs. (25) and (26) for global or local flows in the circular flux surface limit.

In a sample numerical calculation of IBW-driven flows in a TFTR-like plasma, the flows appear to be of a significant size for this direct launch IBW case. The results appear to agree qualitatively with experimental measurements although no attempt at detailed modeling was made. Analytical expressions were obtained in Eqs. (42) - (45) which give the scaling of the expected flows driven by IBW waves in the diffusive limit. 
In conclusion, this paper together with earlier work provides the theoretical tools needed to accurately compute the poloidal and toroidal flows resulting from a given rf scenario. This should be useful in planning future IBW experiments and in carrying out detailed modeling of the results.

\section{Acknowledgments}

The authors wish to thank E.F. Jaeger and L.A. Berry for illuminating discussions. This work was supported by the U.S. Department of Energy (DOE) under grant DEFG03-97ER54392; however, such support does not constitute an endorsement by the DOE of the views expressed herein. 


\section{References}

1 S.C. Chiu and V.S. Chan, Nucl. Fusion 29, 1907 (1989).

2 G.G. Craddock and P.H. Diamond, Phys. Rev. Lett. 67, 1535 (1991).

3 B. LeBlanc, S. Batha, R. Bell, S. Bernabei, et al. Phys. Plasmas 2, 741 (1995).

4 R. Cesario, A. Cardinali, C. Castaldo, M. Leigheb, et al., Phys. Plasmas 8, 4721 (2001).

5 J. D. Moody, M. Porkolab, C. L. Fiore, F. S. McDermott, Y. Takase, J. Terry, and S. M. Wolfe, Phys. Rev. Lett. 60, 298 (1988).

6 M. Ono, P. Beiersdorfer, R. Bell, S. Bernabei, et al., Phys. Rev. Lett. 60, 294 (1988).

7 T. Seki, K. Kawahata, M. Ono, K. Ida, et al., in AIP Conference Proceedings $244-$ Radio Frequency Power in Plasmas, Charleston, SC (American Institute of Physics, New York, 1991), p. 138.

8 B.P. LeBlanc, R.E. Bell, S. Bernabei, J.C. Hosea, et al., Phys. Rev. Lett. 82, 331 (1999);

J.R. Wilson, R.E. Bell, S. Bernabei, K. Hill, et al., Phys. Plasmas 5, 1721 (1998).

9 A. G. Elfimov, G. Amarante Segundo, R. M. O. Galvão, and I. C. Nascimento Phys. Rev. Lett. 84, 1200 (2000).

10 C. Liu and B. Weyssow, Phys. Plasmas 8, 4390 (2001).

11 J.R. Myra and D.A. D’Ippolito, Phys. Plasmas 7, 3600 (2000).

12 J.R. Myra and D.A. D'Ippolito, in AIP Conference Proceedings 595 - Radio

Frequency Power in Plasmas, Oxnard, California (American Institute of Physics, New York, 2001), p. 390.

13 L.A. Berry, E.F. Jaeger and D.B. Batchelor, Phys. Rev. Lett. 82, 1871 (1999).

14 E.F. Jaeger, L.A. Berry and D.B. Batchelor, Phys. Plasmas 7, 641 (2000).

15 E. F. Jaeger, L. A. Berry, and D. B. Batchelor Phys. Plasmas 7, 3319 (2000).

16 E.F. Jaeger, L.A. Berry, E.D. D’Azevedo, D.B. Batchelor and M.D. Carter, Phys. Plasmas 8, 1573 (2001). 
17 J.J. Martinell and C. Gutiérrez-Tapia, Phys. Plasmas 8, 2808 (2001).

18 V.S. Chan and S.C. Chiu, Phys. Fluids B 5, 3590 (1993).

19 N.C. Lee and G.K. Parks, Phys. Fluids 26, 724 (1983).

20 D.A. D’Ippolito and J.R. Myra, Phys. Fluids 29, 2594 (1986).

21 F.W. Perkins, R.B. White and V.S. Chan, Phys. Plasmas 9, 511 (2002).

22 F.W. Perkins, R.B. White, P.T. Bonoli and V.S. Chan, Phys. Plasmas 8, 2181 (2001).

23 V.S. Chan, S.C. Chiu and Y.A. Omelchenko, Phys. Plasmas 9, 501 (2002).

24 C.S. Chang, C.K. Phillips, R. White, S. Zweben et al., Phys. Plasmas 6, 1969 (1999).

25 C.S. Chang, P.T. Bonoli, J.E. Rice and M.J. Greenwald, Phys. Plasmas 7, 1089 (2000).

26 B. Coppi, G. Penn and L.E. Sugiyama, in Proceedings of the 17th International Conference on Fusion Energy, Yokohama, Japan, 1998 (International Atomic Energy Agency, Vienna, 1999), paper IAEA-F1-CN-TH3/7.

27 F.L. Hinton and Y.-B. Kim, Nucl. Fusion 34, 899 (1994).

28 Y.R. Lin-Liu,V.S. Chan and S.C. Chiu, in AIP Conference Proceedings 355 - Radio Frequency Power in Plasmas, Palm Springs, California (American Institute of Physics, New York, 1996), p. 247.

29 V.S. Chan, S.C. Chiu, Y.R. Lin-Liu and Y.A. Omelchenko in AIP Conference Proceedings 485 - Radio Frequency Power in Plasmas, Annapolis, Maryland (American Institute of Physics, New York, 1999), p. 45; and refs. therein.

30 J.W. Connor, S.C. Cowley, R.J. Hastie and L.R. Pan, Plasma Phys. Controlled Fusion 29, 919 (1987).

31 J.R. Myra, Phys. Fluids 31, 1190 (1988).

32 A.B. Hassam and R.M. Kulsrud, Phys. Fluids 21, 2271 (1978).

33 M. Ono, in AIP Conference Proceedings 355 - Radio Frequency Power in Plasmas, Palm Springs, California (American Institute of Physics, New York, 1996), p. 251. 34 E.F. Jaeger and L.A. Berry, private communication, 2002. 


\section{Figure captions}

1. The rf fields for a case with strong IBW absorption at the cyclotron resonance layer: a) rf wave field $\mathrm{E}_{\mathrm{x}}(\mathrm{x})(\mathrm{V} / \mathrm{cm})$; b) absorbed power $\mathrm{P}(\mathrm{x})\left(\mathrm{W} / \mathrm{cm}^{3}\right)$ (solid) and Poynting flux $\mathrm{S}(\mathrm{x})\left(\mathrm{W} / \mathrm{cm}^{2}\right)$ (dashed). The wave is incident from the right, and the resonance is at $\mathrm{x}=0$.

2. Flow velocity and force in the vicinity of the rf absorption layer: a) toroidal, b) poloidal. The forces are shown in arbitrary units. In a), the zero line for the toroidal force is indicated by a dashed horizontal line.

3. Radial profiles of the flows over the entire plasma minor radius: a) toroidal and b) poloidal. 


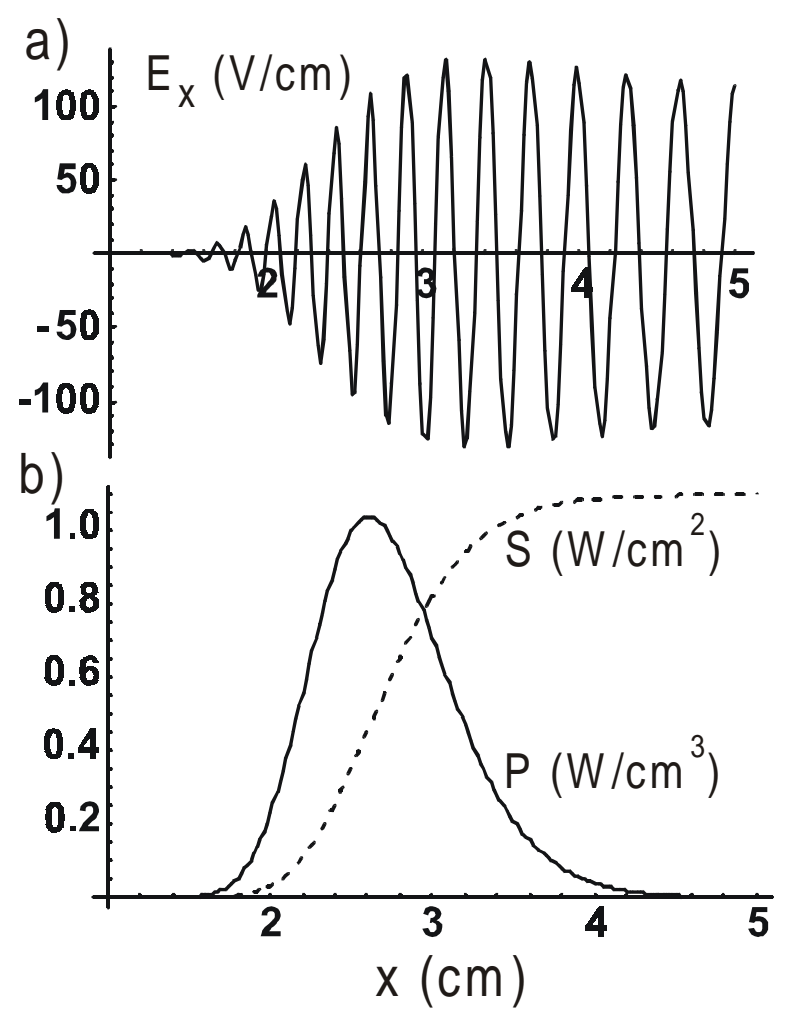

Fig. 1 
a)

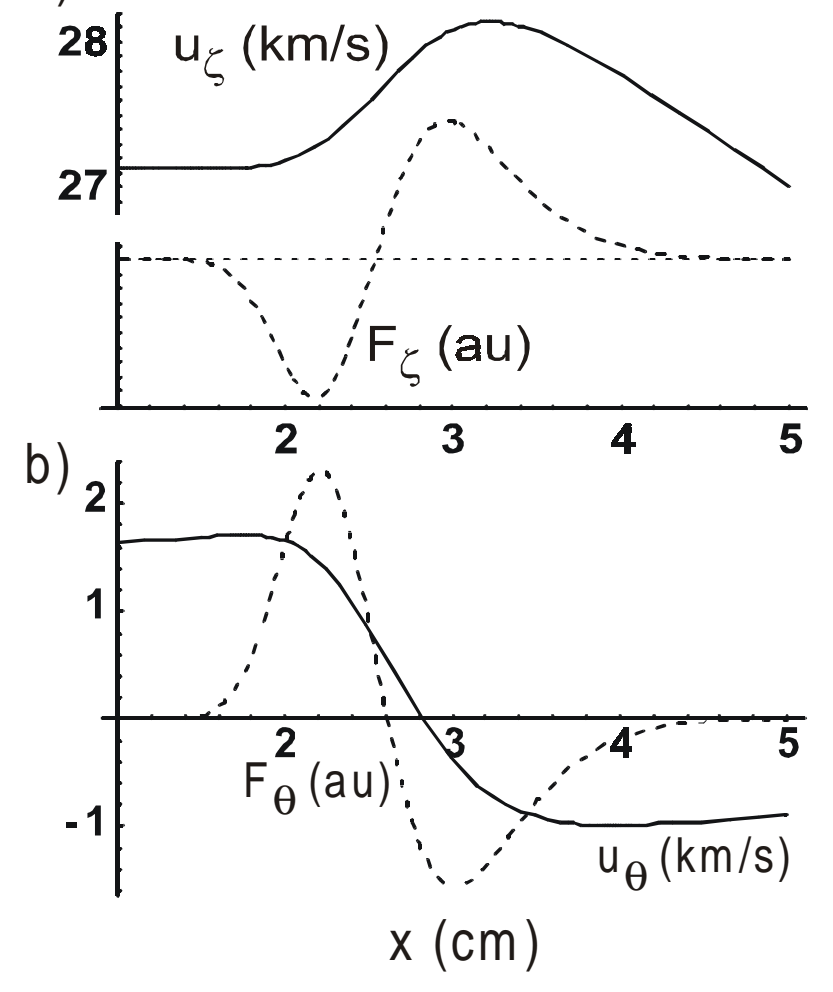

Fig. 2 
a)

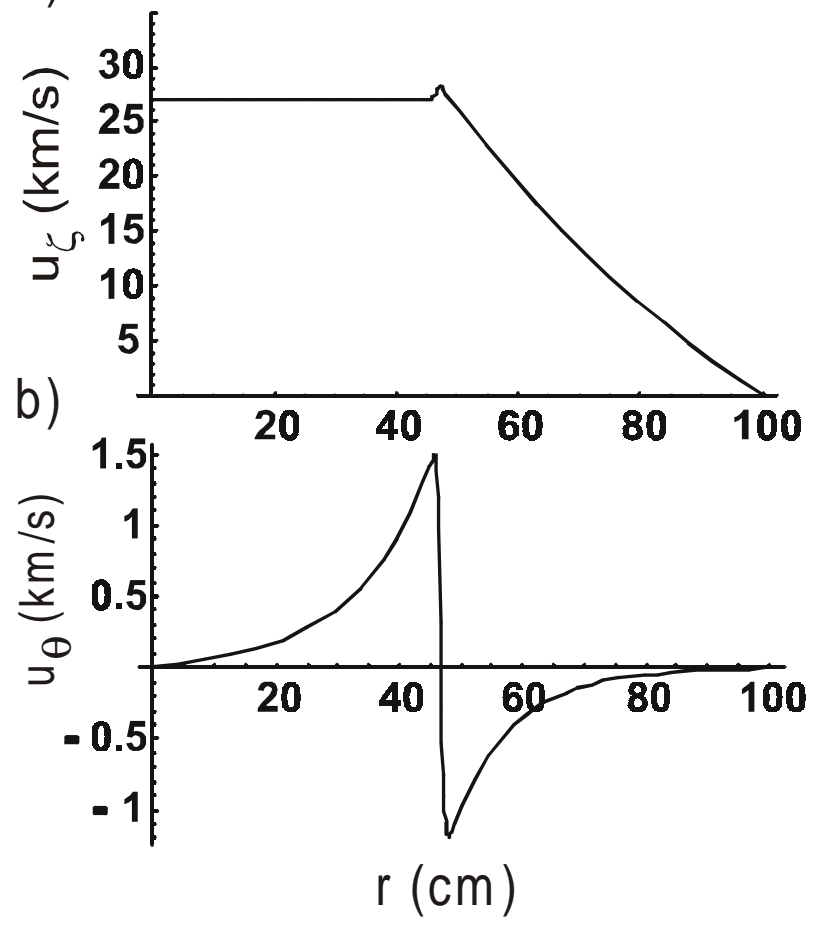

Fig. 3 